\title{
Clinical decision aids for chest pain in the emergency department: identifying low-risk patients
}

This article was published in the following Dove Press journal:

Open Access Emergency Medicine

16 November 2015

Number of times this article has been viewed

\section{William Alley}

Simon A Mahler

Department of Emergency Medicine, Wake Forest Baptist Medical Center, Winston-Salem, NC, USA
Correspondence: William Alley Department of Emergency Medicine, Wake Forest Baptist Medical Center, Medical Center Boulevard, Winston-Salem, NC 27I57, USA Email walley@wakehealth.edu
Abstract: Chest pain is one of the most common presenting complaints in the emergency department, though only a small minority of patients are subsequently diagnosed with acute coronary syndrome (ACS). However, missing the diagnosis has potential for significant morbidity and mortality. ACS presentations can be atypical, and their workups are often prolonged and costly. In order to risk-stratify patients and better direct the workup and care given, many decision aids have been developed. While each may have merit in certain clinical settings, the most useful aid in the emergency department is one that finds all cases of ACS while also identifying a substantial subset of patients at low risk who can be discharged without stress testing or coronary angiography. This review describes several of the chest pain decision aids developed and studied through the recent past, starting with the thrombolysis in myocardial infarction (TIMI) risk score and Global Registry of Acute Coronary Events (GRACE) scores, which were developed as prognostic aids for patients already diagnosed with ACS, then subsequently validated in the undifferentiated chest pain population. Asia-Pacific Evaluation of Chest Pain Trial (ASPECT); Accelerated Diagnostic Protocol to Assess Patients With Chest Pain Symptoms Using Contemporary Troponins (ADAPT); North American Chest Pain Rule (NACPR); and History, Electrocardiogram, Age, Risk factors, Troponin (HEART) score have been developed exclusively for use in the undifferentiated chest pain population as well, with improved performance compared to their predecessors. This review describes the relative merits and limitations of these decision aids so that providers can determine which tool fits the needs of their clinical practice setting.

Keywords: chest pain, decision aid, risk score, acute coronary syndrome

\section{Introduction}

Chest pain is one of the most common presenting complaints in the emergency department (ED), accounting for $9 \%-10 \%$ of annual visits. ${ }^{1}$ While the majority of these cases do not result in a diagnosis of acute coronary syndrome (ACS), missing the diagnosis has potential to result in significant morbidity and mortality. Some estimates suggest double the likelihood of death for patients who are discharged from the ED with myocardial infarction. ${ }^{2}$ Adding complexity to the problem, the symptoms of ACS are frequently atypical, and traditional risk factors for coronary artery disease have little diagnostic utility in the acute care setting. As a result, in order to avoid missing the diagnosis of ACS, the workup for ED patients with acute chest pain is often prolonged and costly.

In an attempt to more accurately risk-stratify patients with chest pain, physicians and researchers have sought out objective tools to better risk-stratify patients; determining who needs further emergent workup and who can be safely discharged home. The ideal risk stratification tool is sensitive and specific enough to identify a subset of 
patients who present with chest pain who can be discharged from the ED after an abbreviated and cost-effective workup, while maintaining an acceptable miss rate for short-term adverse cardiac events. However, the acceptable miss rate for ACS is a matter of considerable debate. In their often cited study from more than a decade and a half ago, Pope et $\mathrm{al}^{2}$ concluded that it would be difficult to achieve a reduction in their reported $2 \%-4 \%$ miss rate for acute myocardial infarction and unstable angina. Kline et $\mathrm{al}^{3}$ calculated that a $2 \%$ miss rate should be acceptable based on the testing threshold at which the risk of harm from further testing equals or exceeds the chance of benefit from confirming ACS. However, the most frequently sited acceptable miss rate is less than $1 \%,{ }^{4}$ so most decision aids aim for a negative predictive value $(\mathrm{NPV})>99 \%$.

This review paper discusses the merits and limitations of validated clinical decision aids used by emergency providers to risk-stratify patients with acute chest pain. This includes discussion of older, commonly used decision aids, such as the thrombolysis in myocardial infarction (TIMI) risk score, Modified TIMI, and Global Registry of Acute Coronary Events (GRACE) score, which were first derived and validated among patients with ACS, and later validated in patients with undifferentiated chest pain. In addition, we discuss newer aids, derived and validated in ED patients with undifferentiated chest pain and designed to identify patients for early discharge from the ED without stress testing or coronary angiography.

\section{Decision aids derived in patients with ACS \\ TIMI risk score}

As treatment modalities for patients with unstable angina and non ST-elevation myocardial infarction were developed and refined, it became necessary to develop a decision aid to help determine which patients were at high risk for adverse outcomes and likely to benefit from aggressive therapy. To meet this need, the TIMI risk score was derived in the late $1990 \mathrm{~s}^{5}$ from the Thrombolysis in Myocardial Infarction 11B trial $^{6}$ and Efficacy and Safety of Subcutaneous Enoxaparin in Unstable Angina and Non-Q-Wave Coronary Event trial. ${ }^{7}$ In each trial, patients were included if they had at least one episode of angina at rest within the preceding 24 hours prior to presentation. In addition, patients had at least one of the following: transient ST-elevation or depression, documented history of coronary artery disease, or elevated serum cardiac biomarkers. The primary end point of the derivation study was major adverse cardiovascular events (MACE), which included all-cause mortality, new or recurrent myocardial infarction, or severe recurrent ischemia prompting urgent revascularization through 14 days. $^{5}$

Seven characteristics were identified as predictors for MACE, each with similar odds ratios, and thus a value of 1 was assigned for each variable for a total possible TIMI risk score of 7 (Figure 1). In the derivation cohort, MACE rates ranged from $4.7 \%$ with a score of 0 or 1 to $40.9 \%$ with a score of 6 or 7 . Validation was then undertaken in three separate cohorts, showing similar rates of increasing risk for adverse outcomes associated with increasing TIMI scores. ${ }^{5}$

The greatest shortcoming of the TIMI risk score derivation and validation studies is that the patient population did not include the majority of patients who present to the ED with chest pain: patients without ischemic electrocardiogram (ECG) changes, no history of coronary artery disease, and normal cardiac biomarkers. Also, the $4.7 \%$ MACE rate among the lowest risk group prevents the TIMI risk score from being used as a tool to identify ED patients for early discharge (discharge without stress testing or angiography).

Although it was not designed for ED use, multiple studies have addressed the utility of the TIMI risk score in ED patients with undifferentiated chest pain. Pollack et $\mathrm{al}^{8}$ demonstrated that the TIMI risk score was predictive of 30 -day adverse outcomes, with approximately 2\% MACE rate among patients with a TIMI risk score of 0 . However, a meta-analysis by Hess et al, ${ }^{9}$ which included 17,265 patients, found the TIMI risk score insufficiently sensitive to be used to identify ED patients for early discharge.

\section{Modified TIMI}

The modified TIMI score ${ }^{10}$ is abbreviated for ease of use in the ED setting. Unlike the TIMI risk score, the modified TIMI score utilizes only four variables: elevated cardiac marker, ischemic ECG changes, age $>65$ years, and history of coronary artery disease. In a retrospective study of 947 ED patients with concern for ACS, the modified TIMI had similar predictive performance compared to the original TIMI risk score. Based on these findings, the authors indicated that their simpler modified score may be more useful in the ED setting than the original TIMI risk score. ${ }^{10}$ However, the modified TIMI risk score also suffers from the same drawbacks as the original TIMI risk score, with a $2.4 \%$ MACE rate even in the lowest risk category and thus is not sensitive enough to be used in isolation as a decision aid to determine which patients are appropriate for early discharge. 


\begin{tabular}{|l|l|l|}
\hline TIMI score & $\begin{array}{c}\text { Yes } \\
\mathbf{1} \text { point }\end{array}$ & $\begin{array}{c}\text { No } \\
\mathbf{0} \text { points }\end{array}$ \\
\hline Age $\geq 65$ & & \\
\hline $\begin{array}{l}\geq 3 \text { risk factors for ACS; hypertension, } \\
\text { hyperlipidemia, smoking, diabetes, family history }\end{array}$ & & \\
\hline Use of aspirin in last 7 days & & \\
\hline Prior coronary stenosis $\geq 50 \%$ & & \\
\hline$\geq 2$ angina events in 24 hours or persisting discomfort & & \\
\hline ST-segment deviation of $\geq 0.05$ mV on initial ECG & & \\
\hline Elevated cardiac biomarkers & & \\
\hline Total score & & \\
\hline Low risk & $0-2$ & \\
\hline Intermediate risk & $3-4$ & \\
\hline High risk & $5-7$ & \\
\hline
\end{tabular}

\begin{tabular}{|l|l|l|l|l|l|l|l|l|l|}
\hline \multicolumn{10}{|l|}{ Grace score } \\
\hline Age & Points & HR & Points & SBP & Points & Cr & Points & Killip class & Points \\
\hline$<39$ & 0 & $<70$ & 0 & $<80$ & 40 & $0.0-0.39$ & 1 & I & 0 \\
\hline $40-49$ & 18 & $70-89$ & 5 & $80-99$ & 37 & $0.4-0.79$ & 4 & II & 15 \\
\hline $50-59$ & 36 & $90-109$ & 10 & $100-119$ & 30 & $0.8-1.19$ & 7 & III & 29 \\
\hline $60-69$ & 55 & $110-149$ & 17 & $120-139$ & 23 & $1.2-159$ & 10 & IV & 44 \\
\hline $70-79$ & 73 & $150-199$ & 26 & $140-159$ & 17 & $1.6-1.99$ & 13 & Cardiac arrest & 30 \\
\hline $80-89$ & 91 & $\geq 200$ & 34 & $160-199$ & 7 & $2.0-3.99$ & 21 & $\begin{array}{l}\text { Elevated } \\
\text { cardiac } \\
\text { markers }\end{array}$ & 13 \\
& & & & & & & 28 & $\begin{array}{l}\text { ST-segment } \\
\text { deviation }\end{array}$ & 17 \\
\hline$>90$ & 100 & - & - & $\geq 200$ & 0 & $\geq 4$ & 28 & $1-88$ \\
\hline
\end{tabular}

Figure I TIMI score and GRACE score.

Abbreviations: ACS, acute coronary syndrome; Cr, creatinine; ECG, electrocardiogram; GRACE, Global Registry of Acute Coronary Events; HR, heart rate; SBP, systolic blood pressure; TIMI, thrombosis in myocardial infarction.

\section{GRACE}

Like the TIMI score, the GRACE score was derived retrospectively from a large cohort of patients with confirmed ACS. ${ }^{11}$ In the derivation study, investigators collected an observational data set on the characteristics and outcomes of patients with ACS from multiple sites in 14 countries. From this large data set, two separate risk stratification scores were derived. The first, developed by Granger et al, ${ }^{12}$ comprised of eight variables and was designed to predict in-hospital mortality. These variables included Killip class, age, blood pressure, resuscitated cardiac arrest, positive cardiac biomarkers, creatinine level, ST-segment shift, and heart rate. Each item was given a weighted score based on its predictive value. In derivation and validation cohorts as well as independent data from GUSTO-IIb, ${ }^{13}$ the GRACE score showed excellent predictive ability for mortality. Patients with scores of $\leq 100$ had $\leq 0.8 \%$ risk of in-hospital mortality, while patients with scores $\geq 250$ had $>50 \%$ risk of mortality.
A second risk score derivation from the GRACE data set, derived by Eagle et al, ${ }^{14}$ was developed to predict 6-month mortality. In this study, nine variables were found to be independently predictive: older age, history of myocardial infarction, history of heart failure, increased pulse rate at presentation, lower systolic blood pressure at presentation, elevated initial serum creatinine level, elevated initial serum cardiac biomarker levels, ST-segment depression on presenting ECG, and not having a percutaneous coronary intervention (PCI) performed in hospital. A weighted value is given to each item, and each item is added to give a total score, which is plotted on a nomogram to estimate the risk of 6-month mortality (Figure 1).

Like the TIMI score, the GRACE score has been validated in an undifferentiated chest pain population. Lyon et $\mathrm{al}^{15}$ calculated a GRACE and TIMI score on 1,000 consecutive patients retrospectively based on chart data, and their outcomes were followed for 30-day MACE. A complete GRACE score could not be calculated on all patients, and some were lost to 
follow-up, leaving 760 patients for final analysis. In this study, patients were grouped by risk score, with the lowest risk group having a $4 \%$ event rate, and the highest risk group, a $71 \%$ event rate. As with TIMI, the GRACE score proved to be predictive of short-term outcomes, but a $4 \%$ event rate in the lowest risk group indicates that the GRACE score is not sensitive enough in the undifferentiated chest pain population to make a decision to discharge a patient without further testing.

While the GRACE scores are predictive of mortality among patients with ACS, they suffer from similar limitations as the TIMI risk score. First, these scores were derived in a confirmed ACS population admitted to the hospital, limiting their generalizability to the undifferentiated ED chest pain population. While they may be useful in helping determine who might benefit from more aggressive therapy, in the immediacy of the ED, that question is usually answered by the presence or absence of ST-elevation and the availability of percutaneous interventional services on-site. The inclusion of PCI as one of the variables for the prediction of 6-month mortality is a major limiting factor for ED use, since most patients seen in the ED with chest pain do not (and should not) receive PCI as part of their evaluation. Finally, both GRACE scores are complex. The weighted scoring for each individual item necessitates the use of a preprinted card or electronic application/calculator. This nonintuitive scoring system limits its utility in a timesensitive acute care setting.

\section{Decision aids developed in an ED chest pain population}

While GRACE and TIMI have gained some traction as risk stratification tools, even in the undifferentiated chest pain patient, it is clear that they are not adequate in isolation to determine which patients can forego further emergent testing. More precise instruments are needed, with several such proposed risk stratification tools developed or derived in the past few years. The decision aids that have shown the greatest promise in identifying a very low-risk cohort of patients eligible for early discharge from the ED will be discussed further.

\section{ASPECT}

The Asia-Pacific Evaluation of Chest Pain Trial (ASPECT) ${ }^{16}$ was one of the earlier attempts to derive a risk stratification tool specifically for undifferentiated chest pain patients in the ED. In 14 EDs across 9 Asian and Pacific countries, patients were evaluated using TIMI score, ECG, and a point-of-care biomarker panel including troponin, creatine kinase $\mathrm{MB}$ (CK-MB), and myoglobin at both presentation and at 2 hours, with the objective of identifying a low-risk cohort that would likely be safe for early discharge.

The primary end point for the study was a composite of death, cardiac arrest, emergency revascularization, cardiogenic shock, ventricular arrhythmia needing intervention, high-degree atrioventricular block needing intervention, or acute myocardial infarction. In all, 3,582 patients were evaluated, with $11.8 \%$ meeting the primary end point. A total of 352 patients $(9.8 \%)$ were deemed low risk, with a TIMI score of 0 , nonischemic ECG, and no evidence of myocardial injury on the biomarker panel (determined by predefined cutoffs for single measures and rate of rise on repeat testing). Of these low-risk patients, only three $(0.9 \%)$ had adverse events. This resulted in a sensitivity of $99.3 \%$ (confidence interval [CI]: 97.9\%-99.8\%), specificity of 11\% (CI: 10.0\%-12.2\%), and NPV of $99.1 \%$ (CI: 97.3\%-99.8\%). ${ }^{16}$

This study demonstrated the feasibility of using a risk stratification decision aid to identify a low-risk group of patients for early discharge. However, it is not without limitations. First, the Asia-Pacific population limits the generalizability of the findings to other regions with different health systems and patient characteristics. Also, this risk stratification tool only identified $9.8 \%$ of patients as low risk for 30-day adverse events, and no mention is made of how many of these patients had follow-up testing or treatment, either inpatient or outpatient, which presumably would have some impact on this number. In practice locations in which providers are highly risk-averse, ASPECT may produce a reduction in the number of patients admitted to the hospital or a chest pain unit for further testing. However, in many EDs, it would actually increase the number of patients identified for further testing. Finally, the use of a point-of-care assay for myoglobin, $\mathrm{CK}-\mathrm{MB}$, and troponin is problematic. The addition of myoglobin and CK-MB to modern troponin assays does not add significant discriminatory value despite increased cost. ${ }^{17}$ Also, point-of-care troponin assays lack sensitivity compared to contemporary central laboratory troponin assays. ${ }^{18}$

\section{ADAPT}

Following ASPECT and as troponin assays became widely available and more sensitive, Than et $\mathrm{al}^{16}$ considered whether a decision aid similar to ASPECT could be used with troponin as the only biomarker to identify patients for early discharge. The ADAPT study ${ }^{19}$ was an observational study evaluating ED patients with chest pain, including those with physician-perceived high risk for having ACS. The risk stratification tool included TIMI score of 0 , nonischemic ECG, and troponin testing at presentation and at 2 hours, 


\begin{tabular}{|c|c|c|c|}
\hline \multicolumn{4}{|l|}{ ADAPT } \\
\hline \multicolumn{2}{|c|}{ High-risk criteria } & Yes & No \\
\hline \multicolumn{4}{|c|}{ 1) $\mathrm{TIMI}$ score $>0$} \\
\hline \multicolumn{4}{|l|}{ a) Age $\geq 65$} \\
\hline \multicolumn{4}{|c|}{ b) $\geq 3$ risk factors } \\
\hline \multicolumn{4}{|c|}{ c) Use of aspirin in last 7 days } \\
\hline \multicolumn{4}{|c|}{ d) Significant coronary stenosis (prior stenosis $\geq 50 \%$ ) } \\
\hline \multicolumn{4}{|c|}{ e) $\geq 2$ angina events in 24 hours or persisting discomfort } \\
\hline \multicolumn{4}{|c|}{ f) ST-segment deviation of $\geq 0.05 \mathrm{mV}$ on initial ECG } \\
\hline \multicolumn{4}{|c|}{ g) Increased initial troponin } \\
\hline \multicolumn{4}{|c|}{ 2) Positive troponin test at 0 or 2 hours } \\
\hline \multicolumn{4}{|c|}{ 3) New ischemic ECG changes } \\
\hline \multicolumn{2}{|l|}{ Heart score } & \multicolumn{2}{|c|}{ Points } \\
\hline \multirow{3}{*}{ History } & Highly suspicious & \multicolumn{2}{|l|}{2} \\
\hline & Moderately suspicious & \multicolumn{2}{|l|}{1} \\
\hline & Slightly suspicious & \multicolumn{2}{|l|}{0} \\
\hline \multirow{3}{*}{ ECG } & Significant ST-depression & \multicolumn{2}{|l|}{2} \\
\hline & Nonspecific repolarization abnormality & \multicolumn{2}{|l|}{1} \\
\hline & Normal & \multicolumn{2}{|l|}{0} \\
\hline \multirow{3}{*}{ Age } & $\geq 65$ & \multicolumn{2}{|l|}{2} \\
\hline & $45-65$ & \multicolumn{2}{|l|}{1} \\
\hline & $\leq 45$ & \multicolumn{2}{|l|}{0} \\
\hline \multirow[t]{3}{*}{ Risk factors } & 3 or more risk factors & \multicolumn{2}{|l|}{2} \\
\hline & $1-2$ risk factors & \multicolumn{2}{|l|}{1} \\
\hline & No risk factors & \multicolumn{2}{|l|}{0} \\
\hline \multirow[t]{3}{*}{ Troponin } & $\geq 3 \times$ normal limit & \multicolumn{2}{|l|}{2} \\
\hline & $1-3 \times$ normal limit & \multicolumn{2}{|l|}{1} \\
\hline & $\leq$ normal limit & 0 & \\
\hline Total & & & \\
\hline
\end{tabular}

\begin{tabular}{|l|l|l|}
\hline NACPR \\
\hline High-risk criteria & Yes & No \\
\hline Age $\geq 50$ & & \\
\hline Acute ischemic ECG changes & & \\
\hline Known coronary artery disease & & \\
\hline Pain typical for ACS & & \\
\hline Any troponin >99th percentile & & \\
\hline
\end{tabular}

Figure 2 ADAPT, NACPR, and the HEART Score.

Notes: ADAPT and NACPR, a patient is considered to be in low risk if they have none of the high-risk criteria. For ADAPT, risk factors include family history of coronary disease, hypertension, hypercholesterolemia, diabetes mellitus, and current smoker. The HEART Score; low risk $=0-3$, high risk $=4$ or greater. Risk factors include currently treated diabetes mellitus, current or recent ( $<90$ days) smoker, diagnosed and/or treated hypertension, diagnosed hypercholesterolemia, family history of coronary artery disease, obesity (body mass index $>30$ ), or a history of significant atherosclerosis (coronary revascularization, myocardial infarction, stroke, or peripheral arterial disease).

Abbreviations: ACS, acute coronary syndrome; ADAPT, Accelerated Diagnostic Protocol to Assess Patients With Chest Pain Symptoms Using Contemporary Troponins; ECG, electrocardiogram; HEART, history, ECG, age, risk factors, troponin; NACPR, North American Chest Pain Rule; TIMI, thrombolysis in myocardial infarction.

and was retrospectively applied to a cohort of 1,975 patients with an overall rate of adverse 30-day outcomes of $15.3 \%$, which was comprised of the composite primary outcome as specified in ASPECT (Figure 2).

This study showed a significant increase in the number of patients deemed low risk and potentially eligible for ED discharge in as little as 2 hours compared to ASPECT, at $20 \%$ vs $9.8 \%$. In this low-risk group, there was only one patient with an adverse event (a myocardial infarction with subsequent revascularization). The tool was $99.7 \%$ sensitive (CI: $98.1 \%-99.9 \%)$ with a $99.7 \%$ NPV (CI: 98.6\%-100.0\%).

The authors also reviewed the rates of follow-up testing, treatment, and interventions for patient in both the high-risk and low-risk groups based on the accelerated diagnostic protocol (ADP). Given the observational design, routine care usually resulted in a significant amount of further testing, with three-quarters of patients in the low-risk group undergoing other investigations, mostly stress testing. Additional treatment occurred in $18.3 \%$, which may have contributed to the sensitivity of the ADP for MACE.
A validation study by Than et $\mathrm{al}^{20}$ in the Asia-Pacific region demonstrated the ability to discharge patients safely with an acceptable miss rate within relatively short ED stays. Likewise, a study by Cullen et $\mathrm{al}^{21}$ in the Asia-Pacific region and Europe, utilizing a modified ADAPT ADP (which included the use of high-sensitivity troponin I measures and a TIMI score of 0 or 1) produced similar results. However, the first validation study in a North American cohort was not as encouraging. ${ }^{22}$ In this secondary analysis of the ACRIN PA4005 $5^{23}$ cohort of 1,140 patients, 551 patients (48.3\%) were identified as low risk by ADAPT. Unfortunately, five of those patients had a 30-day MACE. Therefore, ADAPT correctly identified 26 of 31 patients with MACE, for a sensitivity of $83 \%$ (CI: $66.3 \%-94.5 \%$ ) and NPV of $99.1 \%$ (CI: 97.9\%-99.7\%), far below what is reported in other studies.

In addition, in a recent randomized trial,${ }^{20}$ ADAPT increased the early discharge rate by only $8.3 \%$ compared to usual care. This limitation is likely produced by the use of TIMI score, which classifies patients with aspirin use or two episodes of chest pain in 24 hours as non-low risk. 
Another potential limitation of ADAPT is that some TIMI variables (ie, $\geq 3$ risk factors) can be difficult to accurately ascertain in the ED setting. Given the inconsistent results found in validation studies, the limitations associated with TIMI, and the use of early downstream testing (which may be unavailable in many settings), further study is necessary before ADAPT can be endorsed for widespread use.

\section{North American Chest Pain Rule (NACPR)}

The North American Chest Pain Rule derived by Hess et $\mathrm{al}^{24}$ was developed specifically to address the chest pain population and practice in North America (Figure 2). The rule uses five predictors of risk: ischemic ECG changes not known to be old, history of coronary artery disease, pain typical of ACS, initial and 6-hour troponin greater than 99th percentile, and age greater than 50 years old. Patients without any of these factors were deemed low risk. Further, patients less than 40 years of age only required a single troponin measurement.

In a retrospective observational cohort study of 2,718 patients from 2 Canadian and 1 US ED, NACPR identified $18 \%$ as low risk. Of the $336(12 \%)$ who developed a MACE, none of them were within this low-risk group. This gave the NACPR a sensitivity of 100\% (CI: 97.2\%-100\%), a NPV of $100 \%$ (CI: 99\%-100\%), and a specificity of $20.9 \%$ (CI: 16.9\%-24.9\%). As in the preceding studies, this degree of sensitivity and NPV may be slightly inflated by the testing and treatment given to even the low-risk patients, either inpatient or outpatient. Since this was an observational study, these factors were not controlled for.

The NACPR still requires subsequent validation. However, in one recent study applying the NACPR retrospectively to the MIDAS cohort, ${ }^{25}$ only $4 \%$ of patients were identified as low risk and potentially eligible for early discharge. Application of NACPR in this cohort would have resulted in increased resource utilization, with minimally increased sensitivity for adverse outcomes compared to risk stratification by clinician gestalt.

As with ADAPT, further prospective studies evaluating the NACPR are necessary to determine whether it is a useful tool. One area in particular that bears mentioning is the less than 40-year-old cohort evaluation. Despite a limited evaluation with only the initial troponin as well as the other four predictors in NACPR, Hess et $\mathrm{al}^{9}$ demonstrated 100\% sensitivity for adverse outcomes. Similarly, in a young-patient cohort of 268 patients who had a normal ECG and normal troponin at presentation, there were no adverse cardiac events at 1 year as investigated by Collin et al. ${ }^{26}$ These findings echo commonly observed notions of low cardiovascular risk in patients with young age without concerning findings in the $\mathrm{ED}$, even without further provocative testing.

\section{HEART Score}

The HEART (history, ECG, age, risk factors, and troponin) Score has garnered ample attention recently. Not derived by logistical regression or recursive partitioning multivariate analysis, instead, it was developed simply based on clinical experience and medical literature to determine factors that swayed physicians to admit patients with chest pain to the hospital. The goal of Six et $\mathrm{al}^{27}$ was to develop an easy scoring system akin to the Apgar score. It is made up of five factors: History, ECG, Age, Risk factors, and Troponin. Each factor is scored 0,1 , or 2 , making the scoring system easy to remember and utilize without a computer (Figure 2).

The original study ${ }^{27}$ evaluating the HEART Score spanned over 3 months in a single ED in the Netherlands. A total of 120 patients were enrolled and had follow-up for MACE, a composite outcome of acute myocardial infarction, PCI, coronary artery bypass grafting, or death at 3 months. In this small study, the HEART Score identified roughly onethird of the study cohort as low risk, with only one missed adverse outcome. No analysis was undertaken to evaluate treatment or further testing in the low-risk group, which may have had an effect on this low number.

The first HEART Score validation study by Backus et $\mathrm{al}^{28}$ retrospectively applied the HEART Score to 880 patients from four hospitals in the Netherlands. MACE occurred within 6 weeks in 158 patients (17.95\%). Only $0.99 \%$ of the 303 patients with a HEART Score of $0-3$ had a MACE. This suggests again that roughly one-third of these patients may have been eligible for discharge with minimal risk for adverse outcomes. Further validation studies ${ }^{29,30}$ have shown consistent results in the HEART Score's ability to risk stratify to low or high risk, with less than $2 \%$ MACE rates in those with HEART Score 0-3. However, in many practice settings, including the US, an adverse event rate greater than $1 \%$ is frequently considered unacceptable.

\section{HEART Pathway}

To address the HEART Score's missed MACE rate of $>1 \%$, Mahler et $\mathrm{al}^{31}$ created the HEART Pathway which uses the HEART Score and serial troponins to risk-stratify patients with acute chest pain. To be considered low risk and eligible for early discharge, the HEART Pathway requires a HEART Score of $0-3$ and negative serial troponins. The first study to evaluate the HEART Pathway included 
1,070 patients who were placed into an ED-based observation unit for stress testing. In this cohort, the HEART Pathway was $100 \%$ sensitive (CI: $72 \%-100 \%$ ) with a NPV of $100 \%$ (CI: $94.6 \%-100 \%$ ) for MACE and could have identified $82 \%$ for early discharge (CI: $80 \%-84 \%$ ). Adding reliability to this approach, the authors note that nearly all of the patients in this study received objective testing, onethird with CT coronary angiography. While the HEART Pathway had no cases of missed MACE, use of the HEART Score alone would have missed five patients $(0.6 \%)$ with adverse events.

Validation of the HEART Pathway occurred in a retrospective analysis of data from the MIDAS, a multicenter cohort of 1,107 patients from 18 US ED's with 22\% incidence of ACS. In this study, ${ }^{25}$ the sensitivity of the HEART Pathway for MACE was 99\% (CI: 97\%-100\%) with a NPV of 99\% (CI: 96\%-100\%), while identifying 20\% (95\% CI: $18 \%-23 \%$ ) as low risk and potentially eligible for early discharge. Additional validation occurred in a prospective randomized trial by Mahler et al. ${ }^{32}$ In this study, 282 patients were randomized to the HEART Pathway, combining the HEART Score and troponin measured at 0 and 3 hours, or usual care (based on American College of Cardiology/American Heart Association guidelines). In this study, 39.7\% of patients in the HEART Pathway group were discharged early, compared to $18.4 \%$ in the usual care group. Patients in the HEART Pathway group had a median reduction in hospital length of stay of approximately 12 hours. Importantly, no patients identified as low risk by the HEART Pathway experienced MACE at 30 days.

In addition, this same study specifically evaluated the utilization of objective testing postdischarge. Within 30 days, eight of the low-risk cohort (12\%) received stress testing. In addition, since the HEART Pathway was utilized in this study as a decision aid rather than a substitute for clinical judgment, there were another 19 deemed low risk who received testing during the index visit. Of these two groups, two patients had inducible ischemia on stress testing. One had a follow-up catheterization that showed no CAD and the other was felt to be a false-positive by the cardiologist and did not receive coronary angiography. Neither patient experienced MACE within 30 days.

Based on these studies, the use of the HEART Pathway appears to be safe and efficient, allowing prudent use of resources while minimizing risk of adverse outcomes for patients. Its validation across multiple populations is encouraging, though further randomized controlled trials across diverse populations are necessary.

\section{Conclusion}

Risk stratification decision aids for patients with chest pain have evolved over the past two decades to become more precise and useful in making disposition decisions. Multiple tools have been tested in an undifferentiated chest pain population. TIMI and GRACE were originally derived and validated in patients with confirmed ACS and are best at determining which patients with ACS will benefit from more aggressive therapies. However, they are not sensitive enough to determine which ED patients with undifferentiated chest pain are safe for early discharge without further objective cardiac testing (stress testing or angiography).

In contrast to TIMI and GRACE, ASPECT, ADAPT, the North American Chest Pain Rule, and the HEART Score were developed and validated for use in an undifferentiated ED chest pain population. Few studies have compared these decision aids; however, based on the current evidence, the HEART Score, especially when used in conjunction with serial troponin testing (the HEART Pathway), appears to offer the best combination of sensitivity, NPV, and proportion of patients that can be classified as safe for early discharge. However, the HEART Pathway has yet to be prospectively validated outside of a western European and North American population.

Finally, several new decision aids have been recently developed, and additional decision aids are sure to emerge as knowledge and technology progress. Currently, the new Vancouver Chest Pain Rule, ${ }^{33}$ the Emergency Department Assessment of Chest Pain Score, ${ }^{34}$ and other tools involving advanced imaging modalities are being evaluated or developed. However, at the present time, these decision aids need further validation prior to clinical use.

The holy grail of ED chest pain risk stratification, a decision aid that maximizes the number of patients who can be discharged safely, while catching all cases of near-term adverse cardiovascular events, continues to remain elusive. With the number of chest pain risk scores available, physicians and practices must ask which one is right for their patients. This decision is likely to be based on multiple factors: local practice patterns, test availability, bed space, and risk tolerance of the patient population and physicians. However, great strides have been made over the past two decades. The number of ED patients with acute chest pain who can be identified as having minimal risk for ACS has grown substantially and continues to climb.

\section{Disclosure}

The authors report no conflicts of interest in this work. 


\section{References}

1. Bhuiya FA, Pitts SR, McCaig LF. Emergency department visits for chest pain and abdominal pain: United States, 1999-2008. NCHS Data Brief. 2010;(43):1-8.

2. Pope JH, Aufderheide TP, Ruthazer R, et al. Missed diagnoses of acute cardiac ischemia in the emergency department. $N$ Engl J Med. 2000;342(16):1163-1170.

3. Kline JA, Johnson CL, Pollack CV Jr, et al. Pretest probability assessment derived from attribute matching. BMC Med Inform Decis Mak. 2005;5:26

4. Than M, Herbert M, Flaws D, et al. What is an acceptable risk of major adverse cardiac event in chest pain patients soon after discharge from the Emergency Department? A clinical survey. Int J Cardiol. 2013;166(3): 752-754.

5. Antman EM, Cohen M, Bernink PM, et al. The TIMI risk score for unstable angina/non-ST elevation MI: a method for prognostication and therapeutic decision making. JAMA. 2000;284(7):835-842.

6. Antman EM, McCabe CH, Gurfinkel EP, et al. Enoxaparin prevents death and cardiac ischemic events in unstable angina/non-Q-wave myocardial infarction: results of the thrombolysis in myocardial infarction (TIMI) 11B trial. Circulation. 1999;100:1593-1601.

7. Cohen M, Demers C, Gurfinkel EP, et al. A comparison of lowmolecular-weight heparin with unfractionated heparin for unstable coronary artery disease. N Engl J Med. 1997;337:447-452.

8. Pollack CV Jr, Sites FD, Shofer FS, et al. Application of the TIMI risk score for unstable angina and non-ST elevation acute coronary syndrome to an unselected emergency department chest pain population. Acad Emerg Med. 2006;13:13-18.

9. Hess EP, Agarwal D, Chandra S, et al. Diagnostic accuracy of the TIMI risk score in patients with chest pain in the emergency department: a meta-analysis. CMAJ. 2010;182(10):1039-1044.

10. Jaffery Z, Hudson MP, Jacobsen G, et al. Modified thrombolysis in myocardial infarction (TIMI) risk score to risk stratify patients in the emergency department with possible acute coronary syndrome. J Thromb Thrombolysis. 2007;24(2):137-144.

11. GRACE Investigators. Rationale and design of the GRACE (Global Registry of Acute Coronary Events) project: a multinational registry of patients hospitalized with acute coronary syndromes. Am Heart J. 2001;141:190-199.

12. Granger CB, Goldberg RJ, Dabbous O, et al. Predictors of hospital mortality in the global registry of acute coronary events. Arch Intern Med. 2003;163(19):2345-2353.

13. Cho L, Bhatt DL, Marso SP, et al. An invasive strategy is associated with decreased mortality in patients with unstable angina and non-STelevation myocardial infarction: GUSTO IIb trial. Am J Med. 2003; 114(2):106-111.

14. Eagle KA, Lim MJ, Dabbous OH, et al. A validated prediction model for all forms of acute coronary syndrome: estimating the risk of 6-month postdischarge death in an international registry. JAMA. 2004;291(22):2727-2733

15. Lyon R, Morris AC, Caesar D, et al. Chest pain presenting to the Emergency Department - to stratify risk with GRACE or TIMI? Resuscitation. 2007;74(1):90-93.

16. Than M, Cullen L, Reid CM, et al. A 2-h diagnostic protocol to assess patients with chest pain symptoms in the Asia-Pacific region (ASPECT): a prospective observational validation study. Lancet. 2011;377: 1077-1084.

Open Access Emergency Medicine

\section{Publish your work in this journal}

Open Access Emergency Medicine is an international, peer-reviewed, open access journal publishing original research, reports, editorials, reviews and commentaries on all aspects of emergency medicine. The manuscript management system is completely online and includes a very quick and fair peer-review system, which is all easy to use.
17. Collinson PO, Gaze DC, Thokala P, et al. Randomised assessment of treatment using panel assay of cardiac markers - contemporary biomarker evaluation (RATPAC CBE). Health Technol Assess. 2013; 17(15):1-122.

18. Body R. Acute MI: triple-markers resurrected or Bayesian dice? Lancet. 2011;377:1049-1050.

19. Than M, Cullen L, Aldous S, et al. 2-Hour accelerated diagnostic protocol to assess patients with chest pain symptoms using contemporary troponins as the only biomarker: the ADAPT trial. J Am Coll Cardiol. 2012;59:2091-2098.

20. Than M, Aldous S, Lord SJ, et al. A 2-hour diagnostic protocol for possible cardiac chest pain in the emergency department: a randomized clinical trial. JAMA Intern Med. 2014;174:51-58.

21. Cullen L, Mueller C, Parsonage WA, et al. Validation of high-sensitivity troponin I in a 2-hour diagnostic strategy to assess 30-day outcomes in emergency department patients with possible acute coronary syndrome. J Am Coll Cardiol. 2013;62(14):1242-1249.

22. Mahler SA, Miller CD, Litt HI, et al. Performance of the 2-hour accelerated diagnostic protocol within the American College of Radiology Imaging Network PA 4005 cohort. Acad Emerg Med. 2015;22:452-460.

23. Litt HI, Gatsonis C, Snyder B, et al. CT angiography for safe discharge of patients with possible acute coronary syndromes. $N$ Engl J Med. 2012;366:1393-1403.

24. Hess EP, Brison RJ, Perry JJ, et al. Development of a clinical prediction rule for 30-day cardiac events in emergency department patients with chest pain and possible acute coronary syndrome. Ann Emerg Med. 2012;59:115-125.

25. Mahler SA, Miller CD, Hollander JE, et al. Identifying patients for early discharge: performance of decision rules among patients with acute chest pain. Int $J$ Cardiol. 2013;168:795-802.

26. Collin MJ, Weisenthal B, Walsh KM, et al. Young patients with chest pain: 1-year outcomes. Am J Emerg Med. 2011;29(3):265-270.

27. Six AJ, Backus BE, Kelder JC. Chest pain in the emergency room: value of the heart score. Neth Heart J. 2008;16(6):191-196.

28. Backus BE, Six AJ, Kelder JC, et al. Chest pain in the emergency room: a multicenter validation of the HEART score. Crit Pathw Cardiol. 2010;9:164-169.

29. Backus BE, Six AJ, Kelder JC, et al. A prospective validation of the HEART score for chest pain patients at the emergency department. Int J Cardiol. 2013;168:2153-2158.

30. Six AJ, Cullen L, Backus BE, et al. The HEART score for the assessment of patients with chest pain in the emergency department: a multinational validation study. Crit Pathw Cardiol. 2013;12:121-126.

31. Mahler SA, Hiestand BC, Goff DC, et al. Can the HEART score safely reduce stress testing and cardiac imaging in patients at low risk for major adverse cardiac events? Crit Pathw Cardiol. 2011;10:128-133.

32. Mahler SA, Riley RF, Hiestand BC, et al. The HEART pathway randomized trial: identifying emergency department patients with acute chest pain for early discharge. Circ Cardiovasc Qual Outcomes. 2015;8(2):195-203.

33. Cullen L, Greenslade JH, Than M, et al. The new Vancouver Chest Pain Rule using troponin as the only biomarker: an external validation study. Am J Emerg Med. 2014;32(2):129-134.

34. Than M, Flaws D, Sanders S, et al. Development and validation of the Emergency Department Assessment of Chest Pain Score and $2 \mathrm{~h}$ accelerated diagnostic protocol. Emerg Med Australas. 2014;26(1):34-44.

\section{Dovepress}

Visit http://www.dovepress.com/testimonials.php to read real quotes from published authors. 